\title{
Time Lapse 3D Imaging of Mineral Dissolution
}

CHANDRA WIDYANANDA WINARDHI ${ }^{1}$, JOSE

GODINHO $^{1}$, JENS GUTZMER ${ }^{1}$ AND GERO FRISCH ${ }^{2}$

${ }^{1}$ Helmholtz-Zentrum Dresden-Rossendorf, Helmholtz Institute Freiberg for Resource Technology

${ }^{2}$ Technische Universität Berakademie Freiberg

Presenting Author: c.winardhi@hzdr.de

Kinetics and efficiency of mineral dissolution of multi-phase rocks depend on the properties of the mineral assemblage, the fluid composition and the environment (e.g. temperature, fluid velocity at the surface). Links between the different effects can be studied by reactive transport simulations, although the input of experimentally obtained parameters is not always intuitive and its accuracy depends on the type of experiment. Using gold ore leaching with deep eutectic solvents as an example, we present an in-situ experimental setup to image in $3 \mathrm{D}$ the changes of the solid phases during mineral dissolution using laboratory X-Ray computed tomography (CT). Our new approach allows measuring the volume and surface area at different dissolution times, which are used to calculate the dissolution rate spectra for different mineral phases and to study the kinetics of the dissolution process. Additionally, the 3D model of the dissolving solid imaged by CT allows direct surface extraction for fluid flow simulations, which are used to investigate the effect of fluid velocity on the dissolution rate spectra. Altogether, our method can be used to study complex geochemical mineral - fluid interactions and to directly capture the link between different factors that affect dissolution, e.g.rate spectra and flow, which traditionally can only be studied by an indirect combination of experimental methods and modelling. 